University of South Carolina

Scholar Commons

$12-15-2005$

\title{
Spin-Photovoltaic Effect in Quantum Wires Due to Intersubband Transitions
}

Arkady Fedorov

Yuriy V. Pershin Dr

University of South Carolina - Columbia, pershin@physics.sc.edu

Carlo Piermarocchi

Follow this and additional works at: https://scholarcommons.sc.edu/phys_facpub

Part of the Physics Commons

Publication Info

Published in Physcial Review B, ed. Gene D. Sprouse, Volume 72, Issue 24, 2005, pages

245327-1-245327-8.

Fedorov, A., Pershin, Y. V., \& Piermarocchi, C. (2005). Spin-photovoltaic effect in quantum wires due to intersubband transitions. Physical Review B, 72(24), 245327-1 - 245327-8. DOI: 10.1103/

PhysRevB.72.245327

(c) Physical Review B, 2005, American Physical Society

This Article is brought to you by the Physics and Astronomy, Department of at Scholar Commons. It has been accepted for inclusion in Faculty Publications by an authorized administrator of Scholar Commons. For more information, please contact digres@mailbox.sc.edu. 


\title{
Spin-photovoltaic effect in quantum wires due to intersubband transitions
}

\author{
Arkady Fedorov* \\ Center for Quantum Device Technology, Department of Physics and Department of Electrical and Computer Engineering, \\ Clarkson University, Potsdam, New York 13699-5721, USA \\ Yuriy V. Pershin ${ }^{\dagger}$ and Carlo Piermarocchi ${ }^{\ddagger}$ \\ Department of Physics and Astronomy, Michigan State University, East Lansing, Michigan 48824-2320, USA
}

(Received 19 July 2005; published 21 December 2005)

\begin{abstract}
We consider the current induced in a quantum wire by external electromagnetic radiation. The photocurrent is caused by the interplay of spin-orbit interaction (Rashba and Dresselhaus terms) and an external in-plane magnetic field. We calculate this current using a Wigner functions approach, taking into account radiationinduced transitions between transverse subbands. The magnitude and the direction of the current depend on the Dresselhaus and Rashba constants, strength of magnetic field, radiation frequency, and intensity. The current can be controlled by changing some of these parameters.
\end{abstract}

DOI: 10.1103/PhysRevB.72.245327 PACS number(s): 73.21.Hb, 72.25.Dc, 71.70.Ej, 72.40.+w

\section{INTRODUCTION}

The energy spectrum of free electrons in a perfect quantum wire without a spin-orbit interaction consists of spindegenerate subbands due to the transverse confinement in two directions. In each subband, the energy depends quadratically on the one-dimensional (1-D) momentum. In the presence of an external radiation intersubband excitation probabilities are equal for states with opposite momentum. Therefore, there is no change in current associated with external radiation.

During the last 15 years there has been great interest in theoretical and experimental investigations of photovoltaic effects and photoconductance in quantum wires (see Refs. 1-14 and references therein). Mechanisms for pure spin current generation in 2-D and 1-D systems with a spin-orbit interaction have also been discussed. ${ }^{18,19}$ It is known that a photovoltaic effect is possible in quantum wires without inversion symmetry. For example, a photovoltaic effect in quantum wires with spatially dependent lateral confinement was predicted in Ref. 3. In the present paper we consider a different spin-based mechanism for the photovoltaic effect, which is very interesting in the context of the fast growing field of spintronics. ${ }^{15-17}$ The most important component of our scheme is the spin-orbit interaction. However, the spinorbit interaction alone is not sufficient to generate a charge photocurrent if the quantum wire is spatially homogeneous. Therefore, we consider a wire in an in-plane magnetic field that breaks the inversion symmetry.

We can identify the following groups of intersubband transitions that lead to a photovoltaic effect in quantum wires.

(1) Transitions between spin-splitted subbands with the same confinement quantum numbers.

(2) Transitions between subbands with different confinement quantum numbers.

The main difference between these two groups is that the first is generated by the magnetic field component of electromagnetic radiation, while transitions from the second group are due to the electric field component. In a recent paper ${ }^{13} \mathrm{a}$ spin-photovoltaic effect in quantum wires due to transitions of the first type was considered. It was found that a special role in the photovoltaic effect is played by transitions in which the direction of electron velocity changes. The importance of this velocity inversion was outlined earlier, see, e.g., Ref. 4, in studies of photoconductance. Here we consider intersubband transitions of the second type. An important feature in semiconductor-based quantum wires is that the spin-orbit interaction constants are different for subbands with different confinement quantum numbers. This peculiarity is essential in our scheme for the generation of photocurrent. As we show below, the coupling of an electron to the electric field component of electromagnetic radiation is expected to be much stronger than the coupling to a magnetic component.

Our goal in this paper is to calculate the current in a quantum wire at zero bias voltage due to external radiation. The current as a function of radiation frequency is found numerically from coupled equations involving Wigner functions. The Wigner function formalism has many advantages for investigating transport problems. ${ }^{20-22}$ Among them we mention the phase-space nature of Wigner functions that are similar to the classical Boltzmann distribution functions. This feature makes it possible to separate the incoming and outgoing components of the electron distribution at the boundaries, which, in turn, facilitates the modeling of an ideal contact. The commonly used assumptions are: the distribution of electrons emitted in the quantum wire can be described by the equilibrium distribution function of the leads reservoirs, and all electron are collected by the leads reservoirs without reflection. In this work we extend the description of the transport dynamics to include intersubband transitions due to electromagnetic wave excitation.

We show that the current is sensitive to many control parameters like, e.g., the spin-orbit coupling and external magnetic field. Therefore, the current can be used to determine material parameters. The calculated current strength for a realistic set of parameters is of the order of $0.1 \mathrm{nA}$ and, consequently, can be measured using standard experimental techniques. 


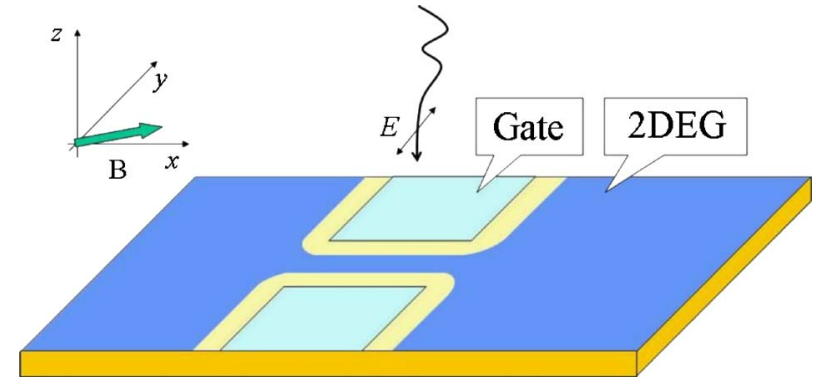

FIG. 1. (Color online) Quantum wire with an applied magnetic field in the $(x, y)$ plane, and irradiated by an electromagnetic wave linearly polarized in the $y$ direction.

This paper is organized as follows. The single-particle energy spectrum and wave functions are introduced in Sec. II. A set of coupled equations for Wigner functions is derived in Sec. III. The discrete model used for numerical solutions is presented in Sec. IV. Numerical results are given and discussed in Sec. V and concluding remarks are in Sec. VI.

\section{SYSTEM}

Figure 1 shows a possible experimental realization of the system under investigation. The two-dimensional electron gas is split into two parts by a potential applied to the gate electrodes. The narrow channel between the gates then forms a quantum wire. Let us define a coordinate system such that the direction of the electron transport through the wire is in the $x$ direction and the lateral confinement is in the $y$ direction. We assume that an external magnetic field is applied in the $(x, y)$ plane. Previously, several interesting investigations of quantum wires with a spin-orbit interaction in the presence of an in-plane magnetic field were reported. ${ }^{23-27}$

In the quantum wire, the Hamiltonian for the conduction electrons can be written in the form

$$
H=H_{0}+H_{1} \text {, }
$$

where

$$
H_{0}=\frac{\mathbf{p}^{2}}{2 m^{*}}-\frac{\alpha}{\hbar} p_{x} \sigma_{y}-\frac{\beta}{\hbar} p_{x} \sigma_{x}+V(y)+U(z)+\frac{g^{*} \mu_{B}}{2} \boldsymbol{\sigma} \mathbf{B},
$$

and

$$
H_{1}=-\frac{e}{m^{*}} \mathbf{A} \mathbf{p}=-\frac{e E_{y} p_{y}}{m^{*} \omega} \cos (\omega t) .
$$

Here, $H_{0}$ is the time-independent part of the Hamiltonian, $H_{1}$ describes the interaction with the electromagnetic field, $\mathbf{p}$ is the momentum of the electron, $m^{*}$ is the effective mass, $V(y)$ is the lateral confinement potential due to the gates, $U(z)$ is the confinement potential in the $z$ direction, $\mu_{B}$ and $g^{*}$ are the Bohr magneton and effective $g$ factor, $E_{y}$ and $\omega$ are the amplitude and frequency of electric field of the polarized electromagnetic wave, and $\boldsymbol{\sigma}$ is the vector of the Pauli matrices. The effect of the external field $\mathbf{B}$ on the spatial motion is neglected, assuming strong confinement in the $z$ direction.
The second and third terms in (1) represent the Rashba and Dresselhaus spin-orbit interaction ${ }^{28,29}$ for an electron moving in the $x$ direction, and $\alpha$ and $\beta$ are the corresponding coupling constants.

The spin-orbit interactions included in the Hamiltonian (2) originate from bulk inversion asymmetry (giving rise to a Dresselhaus interaction ${ }^{29}$ ) and structural inversion asymmetry (giving rise to a Rashba interaction ${ }^{28}$ ). It is well known that the spin-orbit interaction constants are different for electrons in different transverse subbands. ${ }^{18,30}$ In our model we assume that the Rashba spin-orbit interaction constant $\alpha$ depends on the index $n$ and the Dresselhaus spin-orbit interaction constant $\beta$ depends both on $m$ and $n$, where $m$ $=0,1, \ldots$, and $n=0,1, \ldots$, are subband indices due to confinement in the $y$ and $z$ directions, respectively. ${ }^{18,30}$ In the model of rigid quantum wire walls $\beta_{m, n}=\gamma\left\{\left[\pi(n+1) / W_{z}\right]^{2}\right.$ $\left.-\left[\pi(m+1) / W_{y}\right]^{2}\right\}$, where $\gamma$ is a constant.

At $E_{y}=0$, the solutions of the Schrödinger equation can be written in the form

$$
\Psi_{m, n, \pm}(k)=\frac{e^{i k x}}{\sqrt{2}}\left(\begin{array}{c} 
\pm e^{i \varphi_{m, n}} \\
1
\end{array}\right) \phi_{m}(y) \eta_{n}(z)
$$

where

$\varphi_{m, n}=\pi \theta\left(\beta_{m, n} k-\frac{g^{*} \mu_{B}}{2} B_{x}\right)+\arctan \left[-\frac{-\alpha_{n} k+\frac{g^{*} \mu_{B}}{2} B_{y}}{-\beta_{m, n} k+\frac{g^{*} \mu_{B}}{2} B_{x}}\right]$,

$\theta(\cdots)$ is the step function, $\phi_{m}(y)$ and $\eta_{n}(z)$ are the wave function of the transverse modes [due to the confinement potentials $V(y)$ and $U(z)]$. The eigenvalue problem can be solved to obtain

$$
\begin{aligned}
E_{m, n, \pm}(k)= & \frac{\hbar^{2} k^{2}}{2 m^{*}}+\epsilon_{m}+E_{n} \\
& \pm \sqrt{\left(-\alpha_{n} k+\frac{g^{*} \mu_{B}}{2} B_{y}\right)^{2}+\left(-\beta_{m, n} k+\frac{g^{*} \mu_{B}}{2} B_{x}\right)^{2}} .
\end{aligned}
$$

In this expression, $\epsilon_{m}$ and $E_{n}$ are the eigenvalues of decoupled Schrödinger equations in $y$ and $z$ directions. In the experimental setup depicted in Fig. 1, the confinement in the $z$ direction is stronger than the confinement in the $y$ direction, thus we will consider $E_{1}-E_{0} \gg \epsilon_{1}-\epsilon_{0}$.

Recent calculations show that the dependence of the Rashba spin-orbit coupling constant $\alpha$ on $n$ is rather weak. ${ }^{31}$ Therefore, in principle, $\alpha$ could be considered as $n$ independent. However, the particular dependence of $\alpha$ on $n$ is not important for our calculations, since we consider transitions between subbands with $n=0$ only. A useful list of Rashba 

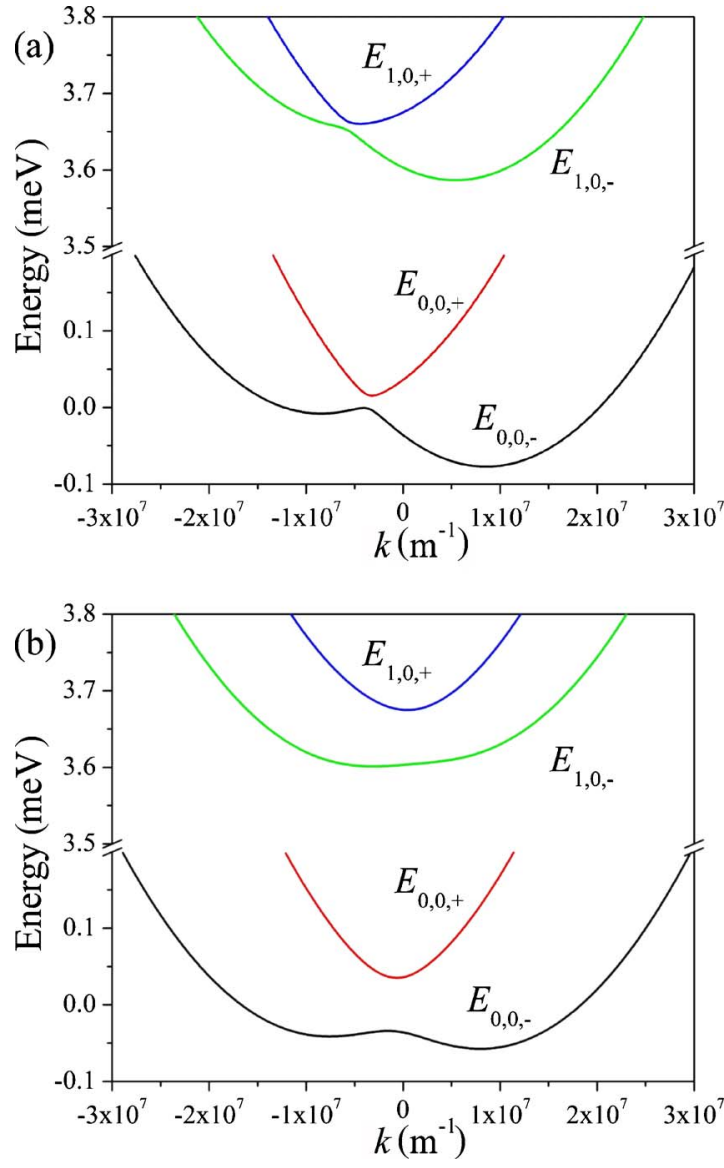

FIG. 2. (Color online) Dispersion relations (two lowest spinsplitted subbands) calculated for (a) $B_{x}=2 \mathrm{~T}, B_{y}=2 \mathrm{~T}$ and (b) $B_{x}$ $=2 \mathrm{~T}, B_{y}=-2 \mathrm{~T}$. These plots were obtained using the parameters values: $m^{*}=0.067 m_{e}, g^{*}=-0.44, \alpha_{0}=0.5 \times 10^{-11} \mathrm{eV} \mathrm{m}, \beta_{0,0}=0.84$ $\times 10^{-11} \mathrm{eV} \mathrm{m}, \beta_{1,0}=0.36 \times 10^{-11} \mathrm{eV} \mathrm{m}, \epsilon_{1}-\epsilon_{0}=3.64 \mathrm{meV}$.

constants in different structures is given in the review paper. ${ }^{32}$ The numerical value $\alpha=0.5 \times 10^{-11} \mathrm{eV}$ m used in our calculations is close to the value $\alpha \simeq 0.4 \times 10^{-11} \mathrm{eV} \mathrm{m}$ experimentally observed in asymmetrically doped $\mathrm{GaAs} / \mathrm{Ga}_{1-x} \mathrm{Al}_{x} \mathrm{As}$ quantum wells. ${ }^{32,33} \mathrm{We}$ would like to emphasize that the mechanism of the photovoltaic effect does not depend on the value of the Rashba constant used by us, but particular dependencies will be different at another value of this parameter.

In what follows we assume that in the absence of radiation the chemical potential in the wire is located between the ground $(0,0)$ and first $(1,0)$ transverse subbands so that only the ground subband is occupied by electrons, and we focus our attention on radiation-induced transitions between these two transverse subbands. The energy spectrum in Eq. (6) is illustrated in Fig. 2 for various directions of $\mathbf{B}$. Notice that the energy dispersion is different (not simply shifted) for the ground and the first transverse subbands. Figure 2 shows that the energy spectrum is strongly asymmetric and significantly dependent on the magnetic field direction. We also note that the gaps between spin-splitted subbands are due to the magnetic field.

The electron velocity is defined by the slope of $E_{m, n, \pm}(k)$ and is given by

$$
\begin{aligned}
v_{m, n, \pm}(k)= & \frac{1}{\hbar} \frac{\partial E_{m, n, \pm}(k)}{\partial k}=\frac{\hbar k}{m^{*}} \\
& \pm \frac{\alpha_{n}\left(\alpha_{n} k-\frac{g^{*} \mu_{B}}{2} B_{y}\right)+\beta_{m, n}\left(\beta_{m, n} k-\frac{g^{*} \mu_{B}}{2} B_{x}\right)}{\hbar \sqrt{\left(-\alpha_{n} k+\frac{g^{*} \mu_{B}}{2} B_{y}\right)^{2}+\left(-\beta_{m, n} k+\frac{g^{*} \mu_{B}}{2} B_{x}\right)^{2}}} .
\end{aligned}
$$

We emphasize that the direction of velocity changes in local extrema points of the spectrum. The radiation-induced transitions that we will consider conserve $k$. Since the positions of local extrema are different in the ground $(0,0)$ and first $(1$, 0) transverse subbands, transitions reversing the velocity direction are possible. The spectrum asymmetry results in asymmetric transition rates and as a result in a finite current at zero bias voltage.

\section{WIGNER FUNCTIONS}

\section{A. Interaction Hamiltonian}

Assuming a parabolic confinement in the $y$ direction we write the ground and the first excited wave functions of the corresponding transverse mode explicitly as

$$
\begin{gathered}
\phi_{0}(y)=\left(\frac{2}{\pi \bar{y}^{2}}\right)^{-1 / 4} \exp \left(\frac{-y^{2}}{\bar{y}^{2}}\right), \\
\phi_{1}(y)=2\left(\frac{2}{\pi \bar{y}^{6}}\right)^{-1 / 4} y \exp \left(\frac{-y^{2}}{\bar{y}^{2}}\right),
\end{gathered}
$$

where $\bar{y}$ is the characteristic width of the quantum wire in the $y$ direction. The energy gap between the ground and the first excited states can be estimated as $\epsilon_{1}-\epsilon_{0}=\left(2 \hbar^{2}\right) /\left(m^{*} \bar{y}^{2}\right)$. Taking the form of the solution for the Schrödinger equation (5) and (8) we obtain the matrix form of the interaction Hamiltonian (3)

$$
H_{1}=i \hbar g\left(e^{i \omega t}+e^{-i \omega t}\right)\left(\sigma^{+} s^{01}-\text { H.c. }\right) \text {, }
$$

where $g=\left(\hbar e E_{y}\right) /\left(2 m^{*} \bar{y} \omega\right)$ is the coupling constant, $\sigma^{+}$is the ladder operator acting on the $y$ component of the wave function: $\sigma^{ \pm} \phi_{m}(y)=\phi_{m \pm 1}(y)$ and $s^{01}$ is an operator in the space of spin degree of freedom,

$$
s^{01}=\left(\begin{array}{ll}
s_{+} & s_{-} \\
s_{-} & s_{+}
\end{array}\right) .
$$

Here $s_{ \pm}=(1 / 2)\left[1 \pm \exp \left(i \Delta \varphi_{n}\right)\right]$ and $\Delta \varphi_{n}=\varphi_{1, n}-\varphi_{0, n}$, and $\varphi_{m, n}$ is defined in Eq. (5). Neglecting high-oscillatory terms we can write the Hamiltonian (9) in the rotating wave approximation $^{34}$ as

$$
H_{1}=i \hbar g\left(e^{-i \omega t} \sigma^{+} s^{01}-\text { H.c. }\right) \text {. }
$$


One can see that for the chosen parameters the coupling constant $g \simeq 10^{9} \mathrm{~s}^{-1}$ is several orders of magnitude larger than the corresponding coupling constant for the interaction with the magnetic component of electromagnetic radiation ${ }^{34}$ $\kappa=\left(\mu_{B} g^{*} / 2\right)\left(n E_{y} / c\right) \simeq 10^{6} \mathrm{~s}^{-1}$, where $n$ is the refractive index of the material and $c$ is speed of light in vacuum. Therefore, we suggest that the photovoltaic effect based on the proposed mechanism is much stronger than that due to the interaction with the magnetic component of the electromagnetic field.

\section{B. Equations for the Wigner functions}

The Liouville-von Neumann equation for the density operator of the electron $\rho_{m m^{\prime}, s s^{\prime}}\left(x, x^{\prime}, t\right)$ is given by

$$
i \hbar \dot{\rho}=[H, \rho],
$$

where $s, s^{\prime}= \pm$ are variables associated with the spin degree of freedom. Henceforth we omit the indices $n, n^{\prime}$ due to con- finement in the $z$ direction since we are interested only in the transitions between the ground $m=0$ and $m=1$ transverse $y$ subbands. The Wigner function can be obtained by integrating ${ }^{35}$

$$
W_{m m^{\prime}, s s^{\prime}}(R, k, t)=\int \rho_{m m^{\prime}, s s^{\prime}}(R, \Delta r, t) \exp (-i k \Delta r) d \Delta r,
$$

where the density operator is written in the new spatial variables $R=\left(r+r^{\prime}\right) / 2$ and $\Delta r=r-r^{\prime}$ and $k$ is the electron wave vector.

Following the standard procedure (see, e.g., Ref. 36) we neglect nonlocal correlations and derive a set of transport equations for Wigner functions in the rotating frame,

$$
\begin{gathered}
\dot{W}_{00,++}+v_{0,+} \frac{\partial W_{00,++}}{\partial x}+\frac{e E_{x}}{\hbar} \frac{\partial W_{00,++}}{\partial k}=g\left[\left(s_{+} W_{10,++}+\text { c.c. }\right)+\left(s_{-} W_{10,-+}+\text { c.c. }\right)\right], \\
\dot{W}_{00,--}+v_{0,-} \frac{\partial W_{00,--}}{\partial x} \frac{e E_{x}}{\hbar} \frac{\partial W_{00,--}}{\partial k}=g\left[\left(s_{-} W_{10,+-}+\text { c.c. }\right)+\left(s_{+} W_{10,--}+\text { c.c. }\right)\right], \\
\dot{W}_{11,--}+v_{1,-} \frac{\partial W_{11,--}}{\partial x}+\frac{e E_{x}}{\hbar} \frac{\partial W_{11,--}}{\partial k}=-g\left[\left(s_{-} W_{10,-+}+\text { c.c. }\right)+\left(s_{+} W_{10,--}+\text { c.c. }\right)\right], \\
\dot{W}_{11,++}+v_{1,+} \frac{\partial W_{11,++}}{\partial x}+\frac{e E_{x}}{\hbar} \frac{\partial W_{11,++}}{\partial k}=-g\left[\left(s_{+} W_{10,++}+\text { c.c. }\right)+\left(s_{-} W_{10,+-}+\text { c.c. }\right)\right], \\
\dot{W}_{01,++}+\frac{v_{0,+}+v_{1,+}}{2} \frac{\partial W_{01,++}}{\partial x}+\frac{e E_{x}}{\hbar} \frac{\partial W_{01,++}}{\partial k}-i\left(\omega_{01,++}-\omega\right) W_{01,++}=g\left[s_{+}\left(W_{11,++}-W_{00,++}\right)+s_{-}\left(W_{11,-+}-W_{00,+-}\right)\right], \\
\dot{W}_{01,+-}+\frac{v_{0,+}+v_{1,-}}{2} \frac{\partial W_{01,+-}}{\partial x}+\frac{e E_{x}}{\hbar} \frac{\partial W_{01,+-}}{\partial k}-i\left(\omega_{01,+-}-\omega\right) W_{01,+-}=g\left[s_{+}\left(W_{11,+-}-W_{00,+-}\right)+s_{-}\left(W_{11,--}-W_{00,++}\right)\right], \\
\dot{W}_{11,-+}+\frac{v_{1,--}+v_{1,+}}{2} \frac{\partial W_{11,-+}}{\partial x}+\frac{e E_{x}}{\hbar} \frac{\partial W_{11,-+}}{\partial k}-i \omega_{11,-+} W_{11,-+}=-g\left[\left(s_{-}^{*} W_{01,++}+s_{+}^{*} W_{01,-+}\right)+\left(s_{+} W_{10,-+}+s_{-} W_{10,--}\right)\right] . \\
\dot{W}_{01,---}+\frac{v_{0,--}+v_{1,-}}{2} \frac{\partial W_{01,--}}{\partial x}+\frac{e E_{x}}{\hbar} \frac{\partial W_{01,---}}{\partial k}-i\left(\omega_{01,---}-\omega\right) W_{01,--}=g\left[s_{+}\left(W_{11,--}-W_{00,--}\right)+s_{-}\left(W_{11,+-}-W_{00,-+}\right)\right], \\
\dot{W}_{01,-+}+\frac{e E_{x}}{\partial x} \frac{\partial W_{00,-+}}{\partial k}-i \omega_{00,-+} W_{00,-+}=g\left[\left(s_{-} W_{10,++}+s_{+} W_{10,-+}\right)+\left(s_{+}^{*} W_{01,-+}+s_{-}^{*} W_{01,--}\right)\right], \\
\frac{v_{01,+}}{2}+\frac{\partial W_{01,-+}}{\hbar} \frac{e E_{x}}{\partial k}-i\left(\omega_{01,-+}-\omega\right) W_{01,-+}=g\left[s_{+}\left(W_{11,-+}-W_{00,-+}\right)+s_{-}\left(W_{11,++}-W_{00,--}\right)\right],
\end{gathered}
$$


For completeness we also included a static electric field $E_{x}$ along the $x$ direction, for example, due to a bias voltage. $\omega_{m m^{\prime}, s s^{\prime}}=\hbar^{-1}\left(E_{m^{\prime}, s^{\prime}}-E_{m, s}\right)$. The left part of the equations (14)-(23) describes the ballistic transport of the electron in the quantum wire, the right part is responsible for excitations induced by the radiation. We consider the "ideal" contact boundary conditions for a wire of length $L$,

$$
\begin{aligned}
& \left.W_{m m, s s}(0, k)\right|_{v_{m, s}(k)>0}=f\left(k, \mu_{l}\right), \\
& \left.W_{m m, s s}(L, k)\right|_{v_{m, s}(k)<0}=f\left(k, \mu_{r}\right),
\end{aligned}
$$

where $f(k, \mu)=1 /\left(1+\exp \left[\left(E_{m, s}(k)-\mu\right) /\left(k_{B} T\right)\right]\right)$ is the Fermi function, $k_{B}$ is the Boltzmann constant, $T$ is the electron temperature, and $\mu_{l / r}$ are chemical potentials of the left/right lead, respectively. We also assume that only the internal part of the quantum wire is irradiated, that is, $E_{y}=0$ for $x<0$ and $x>L$.

The electron charge density, electric charge, and spin currents can be obtained from Wigner functions as

$$
\begin{gathered}
n(x)=\frac{e}{2 \pi} \sum_{m, s} \int_{-\infty}^{\infty} W_{m m, s s}(x, k) d k, \\
I(x)=\frac{e}{2 \pi} \sum_{m, s} \int_{-\infty}^{\infty} v_{m, s}(k) W_{m m, s s}(x, k) d k,
\end{gathered}
$$

and

$$
I_{\gamma}^{s}(x)=\frac{1}{2 \pi} \sum_{m, s} \int_{-\infty}^{\infty}\left\langle\Psi_{m, s}\left|\sigma_{\gamma}\right| \Psi_{m, s}\right\rangle v_{m, s}(k) W_{m m, s s}(x, k) d k,
$$

respectively. Here $\gamma=(x, y, z)$ and different matrix elements can be found in accordance with (5) as $\left\langle\Psi_{m, \pm}\left|\sigma_{x}\right| \Psi_{m, \pm}\right\rangle$ $= \pm \cos \left(\varphi_{m}\right),\left\langle\Psi_{m, \pm}\left|\sigma_{y}\right| \Psi_{m, \pm}\right\rangle=\mp \sin \left(\varphi_{m}\right)$. The details of the numerical model and solution for the derived system of equations are given in the next sections.

\section{DISCRETE MODEL}

The form of Eqs. (14)-(23) does not allow us to solve the problem analytically, even for the stationary case $\partial W / \partial t=0$ and unbiased channel $E_{x}=0$. The solution is complicated by different inter-subband transitions with the change of spin state $s$ originating from the subband asymmetry in the $k$ domain. This effect plays a central role in the electric current generation and should be taken into account. Thus, the system of equations (14)-(23) was solved numerically for $\bar{y}$ $=25 \mathrm{~nm}, \mu=0.0001 \mathrm{eV}$, and $T=0.1 \mathrm{~K}$. We model the domain $x \in[0, L]$ and $k \in\left[-k_{\max }, k_{\max }\right]$ with the mesh sizes of $\Delta x$ $=L /\left(N_{x}-1\right)$ and $\Delta k=2 k_{\max } /\left(N_{k}-1\right)$, respectively. In the calculation we used $N_{x}=25$ and $N_{k}=80$ and the length of the quantum wire $L=2.5 \mu \mathrm{m}$. The value $k_{\max }=3.37 \times 10^{7} \mathrm{~m}^{-1}$ was chosen to ensure that all filled states in $k$ space are taken into account. We fixed the values of diagonal components $W_{m m, s s}$ on the boundaries at $x=0$ for $k$ with $v_{m, s}(k)>0$ and at $x=L$ for $k$ with $v_{m, s}(k)<0$, according to (24) and (25). Simi- larly, for off-diagonal components we fixed the values $W_{m \neq m^{\prime}, s \neq s^{\prime}}=0$ at $x=0$ for $k$ with $\left[v_{m, s}(k)+v_{m^{\prime}, s^{\prime}}(k)\right]>0$ and at $x=L$ for $k$ with $\left[v_{m, s}(k)+v_{m^{\prime}, s^{\prime}}(k)\right]<0$.

The first-order upwind difference scheme was used for the propagation of the Wigner functions in the $\{x, k\}$ domain and second-order two-step Lax-Wendroff scheme ${ }^{37}$ to describe the time-dependent intersubband transfer due to the interaction with the electromagnetic field. The discretized Liouville equation for the Wigner function can be written as

$$
\begin{aligned}
W\left(x_{i}, k_{j}, t_{l+1 / 2}\right)= & W\left(x_{i}, k_{j}, t_{l}\right)-\frac{\Delta t}{2}\left(v\left(k_{j}\right) \frac{\Delta W\left(x_{i}, k_{j}, t_{l}\right)}{\Delta x}\right. \\
& \left.-F\left[W\left(x_{i}, k_{j}, t_{l}\right)\right]\right), \\
W\left(x_{i}, k_{j}, t_{l+1}\right)= & W\left(x_{i}, k_{j}, t_{l}\right)-\Delta t\left(v\left(k_{j}\right) \frac{\Delta W\left(x_{i}, k_{j}, t_{l}\right)}{\Delta x}\right. \\
& \left.-F\left[W\left(x_{i}, k_{j}, t_{l+1 / 2}\right)\right]\right) .
\end{aligned}
$$

where we consider the case $E_{x}=0$ and do not show subband indices for brevity. The upwind/downwind difference is chosen in accordance with

$$
\Delta W\left(x_{i}, k_{j}, t_{l}\right)= \begin{cases}W\left(x_{i}, k_{j}, t_{l}\right)-W\left(x_{i-1}, k_{j}, t_{l}\right), & \text { if } v\left(k_{j}\right)>0, \\ W\left(x_{i+1}, k_{j}, t_{l}\right)-W\left(x_{i}, k_{j}, t_{l}\right), & \text { if } v\left(k_{j}\right)<0 .\end{cases}
$$

The function $F\left[W\left(x_{i}, k_{j}, t_{l}\right)\right]$ embodies the remaining part of Eqs. (14)-(23) which depends only on the Wigner function $W\left(x_{i}, k_{j}, t_{l}\right)$ itself and does not contain partial derivatives. The upwind differencing is stable, ${ }^{37}$ provided the time step is small enough: $\Delta x / \Delta t \leqslant v_{\max }$, where $v_{\max }$ is the maximum possible absolute value of the velocity. Additionally, the time step must be much smaller than the highest frequency of the solution. This condition is satisfied by $\Omega_{R}^{\max } \Delta t \ll 1$, where $\Omega_{R}^{\max }=\max \left[\sqrt{\left(\omega_{m m^{\prime}, s s^{\prime}}-\omega\right)^{2}+4 g^{2}}\right]$ is the maximum possible Rabi frequency involved in the problem. The calculation proved to be stable if these two conditions are met.

The investigation of the effects of the external bias and charge redistribution in the quantum wire is not in the scope of this paper. However, the way to include these effects is straightforward. To consider the effect of voltage applied to the quantum wire, it suffices to add the $x$ component of the electric field $E_{x}[x, V(t)]$ in the discrete model (29). This electric field is a function of the applied potential difference $V(t)=\left(\mu_{r}-\mu_{l}\right) / e$, which can be time dependent and $x$ dependent. The latter is defined by the lead geometry. Additional components of electric field $E_{x}^{q}[n(x)]$ can be calculated self -consistently at each time step to incorporate the effect of charge redistribution.

As was mentioned earlier, transitions between subbands can force the electron to change the direction of the velocity. As a result, the flows of electrons moving in the opposite directions inside the quantum wire intermix, as shown in Fig. 3. Without reflections in the wire, the steady-state solution can be obtained by simply imposing the condition $\partial W / \partial t$ $=0$ and advancing from the given values at left/right bound- 


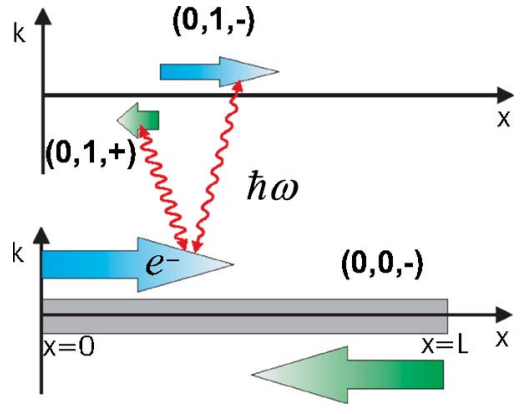

FIG. 3. (Color online) The different subband transition due to the interaction with an electro-magnetic wave are sketched. The interaction causes some electrons to change the direction of the velocity.

aries for $v(k) \gtrless k$. However, in the presence of radiation, the electron distribution at the boundary acquires an additional component, due to the electrons whose velocity direction is changed, and the described above scheme fails. In order to achieve the steady-state solution we considered the temporal dynamics of the system evolving from some initial state until it reached stationary conditions: $\partial W / \partial t \simeq 0$ and $I(x) \simeq I\left(x^{\prime}\right)$ for all $x, x^{\prime} \in[0, L]$. As a initial state we took the values of the Wigner function at equilibrium $W_{m m^{\prime}, s s^{\prime}}(x, k)$ $=\delta_{m m^{\prime}} \delta_{s s^{\prime}} f(k, \mu)$. This corresponds to the uniformly distributed electron density along the channel and is a solution of Eqs. (14)-(23) in the absence of radiation and external bias: $E_{x}=E_{y}=0$. The chosen method of obtaining the steady-state solution provides us also with the transient behavior and, thus, gives more insight into the problem. The drawback is a serious computational effort. The electron distribution reaches the stationary state within the effective time of flight through the quantum wire. Electrons constantly change the direction of velocity due to the interaction with an electromagnetic wave, therefore, this time can be very long compared to the time step $\Delta t$. Fortunately, the slower electrons give the smaller contribution to the current and a steady-state solution can be always found within certain accuracy. The number of steps in time can reach values as much as $N_{t}$ $\sim 10^{5}$. The results of the numerical simulation are presented in Sec. V.

\section{RESULTS AND DISCUSSION}

The photoinduced current through the wire is shown in Fig. 4 as a function of the photon energy. The directions and strength of the magnetic field in this graph are the same as in Fig. 2. The amplitude of the electric field $E_{y}=200 \mathrm{~V} / \mathrm{m}$ used in our calculations was selected close to the electric field amplitude used in recent experiments. ${ }^{38}$ Figure 4 clearly shows a number of current peaks corresponding to different transitions. These peaks depend on the magnetic field strength and direction, as a consequence of the magnetic field dependence of the energy spectrum.

In order to understand transitions leading to a specific peak formation, we consider in detail the current dependence on photon energy for $B_{x}=2 \mathrm{~T}, B_{y}=-2 \mathrm{~T}$ (Fig. 4(b)). It follows from Fig. 4(b) that the current as a function of photon
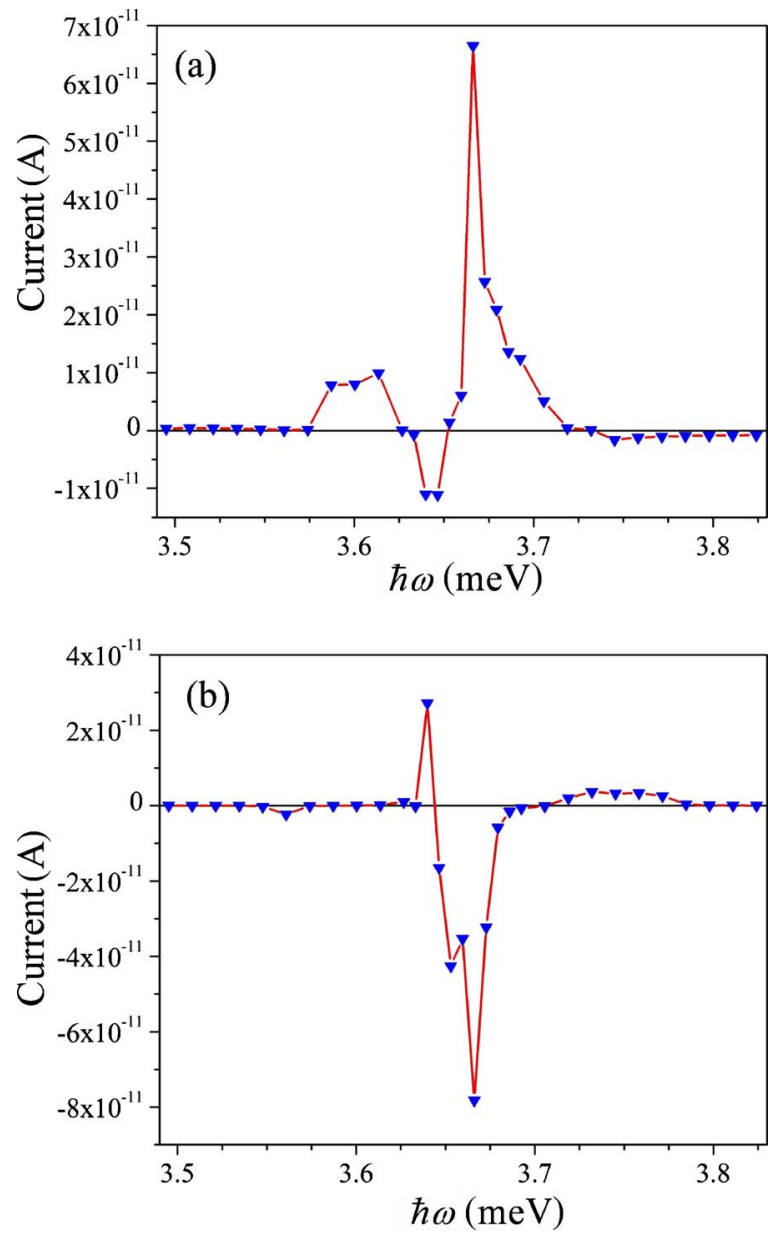

FIG. 4. (Color online) Current through the wire as a function of photon energy $\hbar \omega$ for (a) $B_{x}=2 \mathrm{~T}, B_{y}=2 \mathrm{~T}$ and (b) $B_{x}=2 \mathrm{~T}, B_{y}=$ $-2 \mathrm{~T}$. The parameters values are as in Fig. $2, E_{y}=200 \mathrm{~V} / \mathrm{m}$.

energy has a well pronounced positive peak at $\hbar \omega$ $\simeq 3.64 \mathrm{meV}$, a double negative peak with a minimum at $\hbar \omega \simeq 3.67 \mathrm{meV}$, a small negative peak at $\hbar \omega \simeq 3.565 \mathrm{meV}$, and a broad positive peak of small amplitude at $\hbar \omega$ $\simeq 3.75 \mathrm{meV}$. Figure 5 represents a graphical determination of relevant transitions.

The energy spectrum of two lowest spin-splitted subbands is plotted in the top panel of Fig. 5. The bottom panel of Fig. 5 shows the energy difference between different transverse subbands. By plotting horizontal lines corresponding to the peak energies, in the bottom panel and, by drawing vertical lines through the intersection points of those horizontal lines with energy difference, we can finally identify the points in the top panel corresponding to the peak formation. As it was mentioned above, the important transitions are those that lead to a change of the electron velocity direction. These transitions are shown by arrows in the top panel of Fig. 5 .

In particular, let us consider the large negative peak in the current at $\hbar \omega \simeq 3.67 \mathrm{meV}$ (Fig. 4(b)). Figure 5 shows that the horizontal line $3.67 \mathrm{meV}$ intersects only the $E_{1,0,-}-E_{0,0,-}$ curve in two points. We are reminded that the electron velocity is determined by the slope of $E_{m, n, \pm}(k)$ according to Eq. (7). The left intersection point gives a $k$ vector of transition in which the electron velocity direction is conserved), 


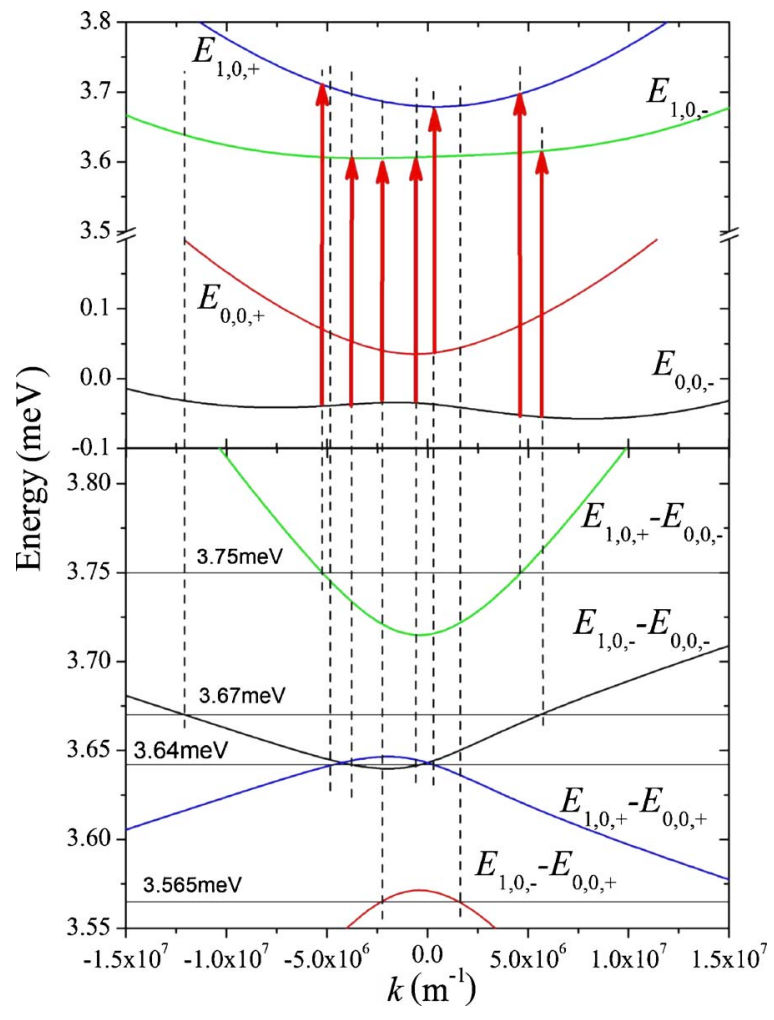

FIG. 5. (Color online) Transitions in the regions of photocurrent peaks for $B_{x}=2 \mathrm{~T}, B_{y}=-2 \mathrm{~T}$. The energy spectrum and difference energies are shown in the top and bottom panels, respectively. Horizontal lines in the bottom panel correspond to excitation energies in peak regions; vertical arrows in the top panel denote transitions when electron velocity direction changes.

because the slopes of $E_{1,0,-}$ and $E_{0,0,-}$ at this value of $k$ are in the same direction. The right intersection point of $3.67 \mathrm{meV}$ line with an $E_{1,0,-}-E_{0,0,-}$ curve gives a transition with a change of electron velocity direction, specifically, with a backscattering of left-moving electrons. Consequently, the electron flux from the right to the left decreases and, because of the negative electron charge, a negative current appears.

Similarly, one can consider transitions at other radiation frequencies. An interesting situation occurs for $\hbar \omega$ $\simeq 3.64 \mathrm{meV}$ excitation, since at this particular frequency three out of four transitions are characterized by the reverse of electron velocity direction. We summarize transitions contributing to the photocurrent at selected radiation frequencies in Table I. The same analysis can also be applied to the result presented in Fig. 4(a), but, because of a more distorted spectrum, the roles of different possible transitions are more dif-

TABLE I. Transitions giving contribution to photocurrent at selected radiation frequencies for $B_{x}=2 \mathrm{~T}, B_{y}=-2 \mathrm{~T}$.

\begin{tabular}{cc}
\hline \hline Excitation energy, meV & Transitions \\
\hline 3.565 & $E_{0,0,+} \rightarrow E_{1,0,-}$ \\
3.64 & $E_{0,0,-} \rightarrow E_{1,0,-} ; E_{0,0,+} \rightarrow E_{1,0,+}$ \\
3.67 & $E_{0,0,-} \rightarrow E_{1,0,-}$ \\
3.75 & $E_{0,0,-} \rightarrow E_{1,0,+}$ \\
\hline \hline
\end{tabular}

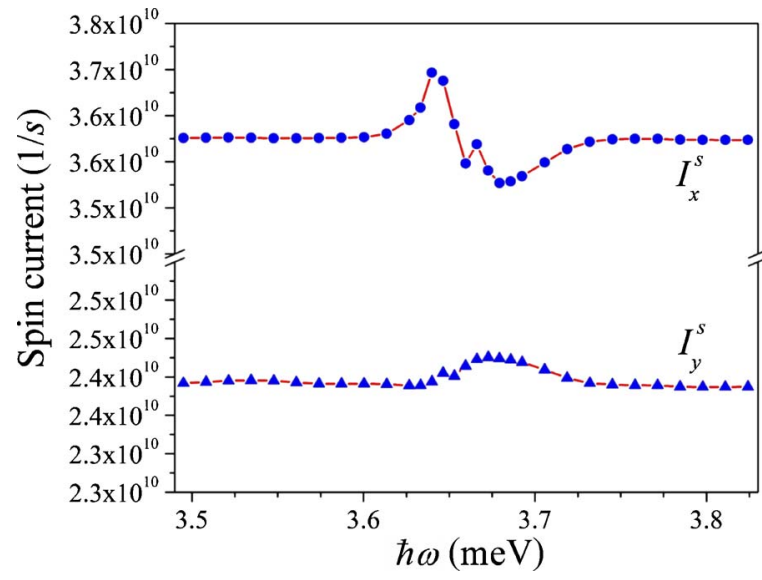

FIG. 6. (Color online) Spin current components for $B_{x}=2 \mathrm{~T}$, $B_{y}=-2 \mathrm{~T}$ at $x=0$.

ficult to interpret. Moreover, we would like to note that, generally, transition probabilities from $E_{0,0,-}$ to $E_{1,0,+}$ and from $E_{0,0,+}$ to $E_{1,0,-}$ subbands are smaller than transition probabilities from $E_{0,0,-}$ to $E_{1,0,-}$ and from $E_{0,0,+}$ to $E_{1,0,+}$ subbands because of the different spin direction in initial and final states. This results in a smaller current peaks at $\hbar \omega$ $\simeq 3.565 \mathrm{meV}$ and $\hbar \omega \simeq 3.75 \mathrm{meV}$ in Fig. 4(b).

As electrons carry spin as well as charge, the external radiation also changes the spin current through the wire. Notice that even without the radiation, the spin current is not zero, due to the spin-orbit interaction. Figure 6 shows spin current components at $x=0$ for $B_{x}=2 \mathrm{~T}, B_{y}=-2 \mathrm{~T}$. We note that the spin current dependence on the radiation frequency has features similar to the charge current (Fig. 4(b)). However, we found that radiation-induced changes in spin current are much less than the equilibrium spin current in the wire. From an experimental point of view, the spin currents are not so easy detectable. Therefore, the observation of this photovoltaic effect through spin current seems unpractical.

\section{CONCLUSIONS}

In summary, in this paper we have investigated the photovoltaic effect in quantum wires with a spin-orbit interaction and an in-plane magnetic field. We have found that the peculiarities of the energy spectrum lead to a photocurrent generation. The dependence of the photoinduced current on the excitation frequency was calculated numerically using the Wigner functions formalism. A system of coupled equations for the Wigner functions was derived and solved numerically for "ideal" contact boundary conditions. We used the firstorder upwind differencing for the propagation in the spatial domain and the second-order two-step Lax-Wendroff differencing for time-dependent inter-subband transitions due to electromagnetic wave excitation. Stable numerical solutions were found under appropriate choices of the time step $\Delta t$. The calculations can be extended to introduce the effects of an external bias and self-consistent potentials due to charge density redistributions, which can be a topic for a future investigation. The frequency dependence of the photoinduced current consists of a set of peaks related to transitions 
between different points of the spectrum. Therefore, the energy spectrum can be reconstructed from photocurrent measurements. Material parameters, such as spin-orbit coupling constants, can be obtained from the analysis of the photocurrent.

\section{ACKNOWLEDGMENTS}

We gratefully acknowledge useful discussions with M. Cheng, L. Fedichkin, and V. Privman. This research was supported by the National Science Foundation, Grants No. DMR-0121146 and DMR-0312491.
*Electronic address: fedorov@ clarkson.edu

$\dagger$ Electronic address: pershin@pa.msu.edu

Electronic address: piermaro@msu.edu

${ }^{1}$ F. Hekking and Yu. V. Nazarov, Phys. Rev. B 44, 11506 (1991).

${ }^{2}$ S. Feng and Q. Hu, Phys. Rev. B 48, 5354 (1993).

${ }^{3}$ L. Fedichkin, V. Ryzhii, and V. Vyurkov, J. Phys.: Condens. Matter 5, 6091 (1993).

${ }^{4}$ A. Grincwajg, L. Y. Gorelik, V. Z. Kleiner, and R. I. Shekhter, Phys. Rev. B 52, 12168 (1995).

${ }^{5}$ F. A. Maao and L. Y. Gorelik, Phys. Rev. B 53, 15885 (1996).

${ }^{6}$ C. S. Tang and C. S. Chu, Phys. Rev. B 53, 4838 (1996).

${ }^{7}$ S. Blom, L. Y. Gorelik, M. Jonson, R. I. Shekhter, A. G. Scherbakov, E. N. Bogachek, and U. Landman, Phys. Rev. B 58, 16305 (1998).

${ }^{8}$ Y. Levinson and P. Wölfle, Phys. Rev. Lett. 83, 1399 (1999).

${ }^{9}$ C. Niu and D. L. Lin, Phys. Rev. B 62, 4578 (2000).

${ }^{10}$ S. Blom and L. Y. Gorelik, Phys. Rev. B 64, 045320 (2001).

${ }^{11}$ G. Platero and R. Aguado, Phys. Rep. 395, 1 (2004).

${ }^{12}$ N. G. Galkin, V. A. Margulis, and A. V. Shorokhov, Phys. Rev. B 69, 113312 (2004).

${ }^{13}$ Yu. V. Pershin and C. Piermarocchi, Appl. Phys. Lett. 86, 212107 (2005)

${ }^{14}$ H. Kosaka, D. S. Rao, H. D. Robinson, P. Bandaru, T. Sakamoto, and E. Yablonovitch, Phys. Rev. B 65, 201307(R) (2002).

${ }^{15}$ Semiconductor Spintronics and Quantum Computation, edited by D. D. Awschalom, N. Samarth, and D. Loss (Springer-Verlag, Berlin, 2002).

${ }^{16}$ I. Žutić, J. Fabian, and S. Das Sarma, Rev. Mod. Phys. 76, 323 (2004).

${ }^{17}$ S. Saikin, Yu. V. Pershin, and V. Privman, IEE Proc.: Circuits Devices Syst. 152, 366 (2005).

${ }^{18}$ E. Ya. Sherman, Ali Najmaie, and J. E. Sipe, Appl. Phys. Lett.
86, 122103 (2005).

${ }^{19}$ S. A. Tarasenko and E. L. Ivchenko, JETP Lett. 81, 231 (2005).

${ }^{20}$ N. C. Kluksdahl, A. M. Kriman, D. K. Ferry, and C. Ringhofer, Phys. Rev. B 39, 7720 (1989).

${ }^{21}$ W. R. Frensley, Phys. Rev. B 36, 1570 (1987).

${ }^{22}$ L. Fedichkin and V. V'yurkov, Appl. Phys. Lett. 64, 2535 (1994).

${ }^{23}$ J. A. Nesteroff, Yu. V. Pershin, and V. Privman, Phys. Rev. Lett. 93, 126601 (2004).

${ }^{24}$ P. Středa and P. Šeba, Phys. Rev. Lett. 90, 256601 (2003).

${ }^{25}$ Yu. V. Pershin, J. A. Nesteroff, and V. Privman, Phys. Rev. B 69, 121306(R) (2004).

${ }^{26}$ R. G. Pereira and E. Miranda, Phys. Rev. B 71, 085318 (2005).

${ }^{27}$ L. Serra, D. Sánchez, and R. López, cond-mat/0504578 (unpublished).

${ }^{28}$ Yu. A. Bychkov and E. I. Rashba, JETP Lett. 39, 78 (1984).

${ }^{29}$ G. Dresselhaus, Phys. Rev. 100, 580 (1955).

${ }^{30}$ S. Pramanik, S. Bandyopadhyay, and M. Cahay, IEEE Trans. Nanotechnol. 4, 2 (2005).

${ }^{31}$ E. Shafir, M. Shen, and S. Saikin, Phys. Rev. B 70, 241302(R) (2004).

${ }^{32}$ R. H. Silsbee, J. Phys.: Condens. Matter 16, R179 (2004).

${ }^{33}$ B. Jusserand, D. Richards, G. Allan, C. Priester, and B. Etienne, Phys. Rev. B 51, R4707 (1995).

${ }^{34}$ W. H. Louisell, Quantum Statistical Properties of Radiation (Wiley, New York, 1973).

${ }^{35}$ E. Wigner, Phys. Rev. 40, 749 (1932).

${ }^{36}$ S. Saikin, J. Phys.: Condens. Matter 16, 5071 (2004).

${ }^{37}$ D. A. Anderson, J. C. Tannehill, and R. H. Pletcher, Computational Fluid Mechanics and Heat Transfer (Hemisphere, New York, 1984).

${ }^{38}$ R. G. Mani, J. H. Smet, K. von Klitzing, V. Narayanamurti, W. B. Johnson, and V. Umansky, Nature (London) 420, 646 (2002). 